\title{
A PROPOSAL FOR A SYSTEMATIZATION AND TAXONOMY OF METHODS TO RECTIFY THERMALLY INDUCED ERRORS ON EXISTING MACHINE TOOLS
}

\author{
C. Gißke ${ }^{1 *}$, T. Albrecht ${ }^{2}$, H. Wiemer ${ }^{2}$, W. Esswein ${ }^{1}$, S. Ihlenfeldt ${ }^{2}$ \\ ${ }^{1}$ TU Dresden, Chair of Business Informatics, esp Systems Engineering, Dresden, Germany \\ ${ }^{2}$ TU Dresden, Institute of Mechatronic Engineering Dresden, Dresden, Germany \\ ${ }^{\star}$ Corresponding author; e-mail: c.gisske@tu-dresden.de
}

\begin{abstract}
In most sectors of today's industry, there is the requirement to manufacture work pieces with accuracy in micron range. However, maintaining this accuracy can be considerably impeded by thermally induced displacements which arise in the production process. Thermally induced errors cause large parts of residual machining errors on modern machine tools. Using climate control systems for whole workshops can counteract these errors. Yet, this method is extremely cost and energy intensive.

To increase machine accuracy and meet the industrial demands in a more efficient way, research offers various methods to minimize this error. These methods differ greatly in their approaches and requirements. Some intervene in the machine structure, while others are based on thermomechanical models and need to be integrated into the software of the control system as correction algorithms. Since machine tools also vary in their kinematic structure and complexity, it is difficult for potential users to select suitable solutions and estimate the effort required to implement them with the available resources. This paper presents a systematization and taxonomy of such methods, which was elaborated based on solutions developed in the project CRC/TR 96. By conducting semi-structured expert interviews, the functional principle, prerequisites and resources required for the application of each solution were recorded, categorized and evaluated in terms of their effort. Based on the presented systematization, it is possible to compare these different methods and evaluate them regarding their implementation effort and flexibility. This is the first step towards a user-specific evaluation of these methods in the future and towards facilitating the transfer of this fundamental research into industrial application.
\end{abstract}

Keywords:

Thermal effects; Thermal error; Correction; Compensation; System

\section{INTRODUCTION}

Despite thermal effects already being identified as a relevant source of machining errors on workpieces in the early 1960s, it was no relevant topic in the industry until the early 1990s [Bryan 1967, Bryan 1990]. Since then, the subject has gained more significance and attention [Mayr 2012]. As stated by Wegner et al., the approach to master thermal errors in machine tools changed from energyintensive cooling strategies and warm-up cycles to more energy-efficient countermeasures [Wegener 2016]. Such countermeasures range from design measures that aim to minimize thermal induced deformation to model-based approaches correcting the displacement of the TCP. Since the 1990s, a large variety of methods and approaches was developed in research to predict and correct the thermal induced deformation of machine tools [Weck 1995, Mayr 2017]. However, the old and energy-intensive approaches of production facilities to use hall air conditioning and warmup cycles are still the dominant measures among machine tool users to minimize thermally induced deformation
[Putz 2018]. This shows that the transfer of newer methods from research to industry seems to be difficult.

While manufacturers of machine tools have the possibility of incorporating solutions that can minimise the thermal displacement error in the machine tool's manufacturing process, this is more difficult to implement for machine tool users. This is due to the fact that such methods usually require retrofitting of components on the existing machine or intervention in the machine control system. Yet, modern control systems theoretically allow a retrofit of these solutions for already existing industrial machines. Thus, the circle of potential users includes not only the manufacturers of machine tools but also their users. However, it can be difficult for a user to decide on a method, as the methods, which are very specific in their nature, require special knowhow, are usually associated with high implementation costs, which are different for each method, and are also different in their effects. Therefore, a case-specific evaluation of the methods is often challenging and very complex. 
This paper attempts to answer the following research question: Is it possible to develop a taxonomy for methods rectifying thermally induced errors on machine tools that allows a comparative evaluation of these methods by establishing a comparable classification system?

To investigate this, nine methods, which are currently being developed in the project CRC/TR 96, were examined with regard to technical and economic aspects in order to be able to create the most comprehensive picture possible of the different methods and to identify similarities and differences, which in turn will enable comparability between them. The methods of the project are well suited for this purpose, as each is different in character and covers a broad spectrum of the initially listed forms of correction and compensation methods as some of them use external components, some are purely simulation-based and some combine these approaches. The function of the investigated methods is briefly explained in Table 1. A detailed description is unfortunately not possible within the scope of this paper.

Table 1 - Approaches of the investigated methods [Großmann 2015]

\begin{tabular}{|c|c|}
\hline Method 1 & $\begin{array}{l}\text { Constructive approach that influences the } \\
\text { heat flow in the machine tool to achieve a } \\
\text { uniform temperature field throughout the } \\
\text { whole structure }\end{array}$ \\
\hline Method 2 & $\begin{array}{l}\text { Parameter-based relation between tool } \\
\text { temperature and resulting tool and chunk } \\
\text { deformation. Correction is achieved via } \\
\text { machine control. }\end{array}$ \\
\hline Method 3 & $\begin{array}{l}\text { Camera-based measurement method to } \\
\text { determine deformation of the machine tool } \\
\text { structure and to correct it via machine } \\
\text { control. }\end{array}$ \\
\hline Method 4 & $\begin{array}{l}\text { Controllable cooling strategy suited to the } \\
\text { activity of the heat sources in the machine } \\
\text { tool to achieve a uniform temperature field }\end{array}$ \\
\hline Method 5 & $\begin{array}{l}\text { Optimization of spindle cooling through } \\
\text { variable, demand-adapted cooling flowl } \\
\text { and optimized cooling channel geometries }\end{array}$ \\
\hline Method 6 & $\begin{array}{l}\text { Correction method of TCP displacement } \\
\text { based on the measured values of special } \\
\text { displacement sensors placed on crucial } \\
\text { structural elements of the machine tool. } \\
\text { From the sensor signals correction values } \\
\text { are calculated and implemented via } \\
\text { machine control. }\end{array}$ \\
\hline Method 7 & $\begin{array}{l}\text { By measuring the TCP displacement } \\
\text { during a specified load case, a relation to } \\
\text { the load torque of the axis drives is } \\
\text { established. From this relation, correction } \\
\text { values are calculated for a correction via } \\
\text { the machine tool control. (grey box model) }\end{array}$ \\
\hline Method 8 & $\begin{array}{l}\text { Full mechanical thermal modelling of the } \\
\text { machine structure enables simulation of } \\
\text { the resulting TCP displacement based on } \\
\text { the motion specifications. Correction }\end{array}$ \\
\hline
\end{tabular}

\begin{tabular}{|l|l|}
\hline & $\begin{array}{l}\text { values are calculated and transferred to } \\
\text { the control unit. (white box model) }\end{array}$ \\
\hline Method 9 & $\begin{array}{l}\text { Characteristic diagrams relate TCP } \\
\text { displacement to measured temperatures } \\
\text { at selected locations on the machine } \\
\text { structure. This is based on extensive } \\
\text { measurements or simulations under } \\
\text { various load scenarios. (black box model) }\end{array}$ \\
\hline
\end{tabular}

\section{METHODOLOGY}

In order to create a categorisation, taking into account both technical and economic aspects, the following aspects were initially assessed as relevant: the implementation effort of the individual methods, the expected impact, any risks to be expected and long-term behaviour. Since all the methods of the project to be examined are still in the development phase and have not yet been transferred into practice, the developers of the individual methods were interviewed as experts on the named aspects in a semi-structured interview, since only they have the specific knowledge on the current status of the methods to gain in-depth insights into the methods.

The experts were first asked about the general characteristics of their method and then asked to describe the work steps and resources required to carry out the method. Subsequently, the respondents were asked to use a questionnaire to estimate the expected effort based on the life cycle costs of the VDMA 34160 guideline, to obtain an estimate of the effort required along the life cycle of the method [VDMA 2006].

The results of the interviews were analysed qualitatively according to the deductive category formation of Mayring [2014]. Deductive qualitative content analysis is used to order, categorise and structure the content of e.g. interviews by using pre-existing categories. Aspects that emerged during the evaluation, which were not previously considered, were inductively added as new categories [Mayring 2014]. In addition, the described work steps were modelled as a process flow using the semi-formal modelling language BPMN. This was done in order to, on the one hand, obtain an overview of the required process steps over the entire life cycle of each method. On the other hand, the modelled process steps make it possible to link resources and potential risks directly to each process step. BPMN was used as it is widely accepted in the industry [Kirchner 2014] and there is already work that has been done on extending the standard language to include the mapping of resources that can be re-used for this modelling purpose [Braun 2014]. An example of the modelling is given in Figure 1. It can be seen that different professions are required for the first process step and that different risks with different probabilities of occurrence are linked to the step. In the second process step, in addition to the need for an engineer, the necessary material use of thermal sensors was also illustrated. In this way, the estimated effort was made comprehensible and could be compared with the subjective assessment of the VDMA life cycle costs.

The categories derived from the results of this evaluation can be used as criteria on the basis of which the various methods can be compared with each other. The criteria are explained in Chapter 3. 


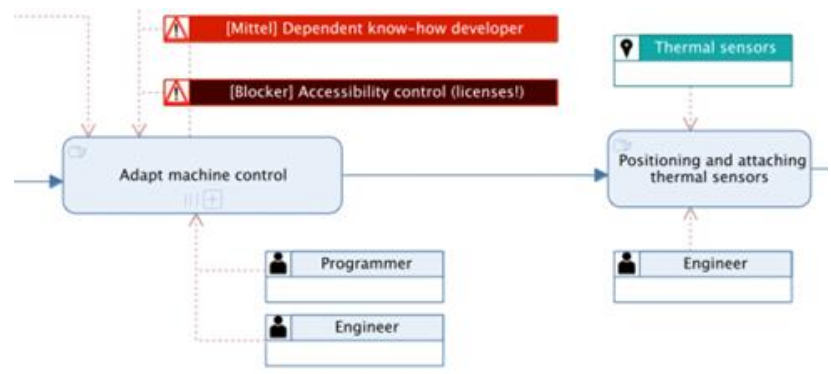

Fig. 1: Example of a modelled process flow with attached resources and risks.

\section{SYSTEMATIZATION OF SOLUTION METHODS}

Figure 2 gives an overview of all of the derived categories. First, general properties of the solutions were identified.

Thus, it can be distinguished whether the method is constructive, metrological or simulation-based.

Constructive methods aim to compensate the heat flow in the machine so that large temperature gradients and resulting displacements are avoided. Solutions that work according to that principle can be called compensation methods.

Metrological and simulation-based methods, on the other hand, aim to predict the amount and direction of the thermally induced deviation at the TCP. The result is provided as a correction value for the machine controls. Methods that work according to this principle can be classified as correction methods.

This distinction follows the understanding of Großmann et al. [Großmann 2015].

From the perspective of retrofitting this solution to existing machine tools, one criterion for comparison can already be derived from this distinction, the degree of invasiveness.

This can be divided into an intervention in the machine structure and the intervention in the machine control.

Both features bring different requirements.

An intervention in the machine structure represents a permanent structural change of the machine. This often represents a design challenge, since additional elements have to be introduced into the limited installation space without impeding the functionality of the machine.

The intervention in the machine control is different depending on the control software. With many established suppliers, it is only possible to add correction values to the position of the machine by purchasing additional functionalities. If these are not available already, this means an additional investment.

The metrological and simulation-based solutions can be divided into white box, grey box or black box models.

Black box models for the correction of thermal displacements establish a functional relationship between input variables such as motor currents or measured values from temperature sensors and the resulting displacement at the TCP. To parameterize this functional relationship for a concrete machine tool, typically load cases adapted to later applications are executed and the displacement of the TCP is determined. This can be realized for example by measurements.

Due to the complex character of the thermal behaviour of machine tools, it cannot be assumed that the black box model will provide correct results if the load deviates significantly from the initial load case.

Since a white box model represents the complete machine behaviour from the heat source to the resulting displacement at the TCP, it can be assumed that the model provides correct results for the respective machine independent of the load case.

For grey box models this is only partly the case.

The flexibility of the solution can be derived from this behaviour as a further criterion. Black box models parameterized by load cases cannot operate outside the range represented by these initial load cases. Therefore they can be seen as load case specific. Whereas a white box model of a whole machine tool can calculate the thermal displacement for every state of the machine. Those approaches can be seen as machine specific. A Grey Box solution, whose functionality can be assumed to be at least partially beyond the initial load cases, is conditionally load case-specific.

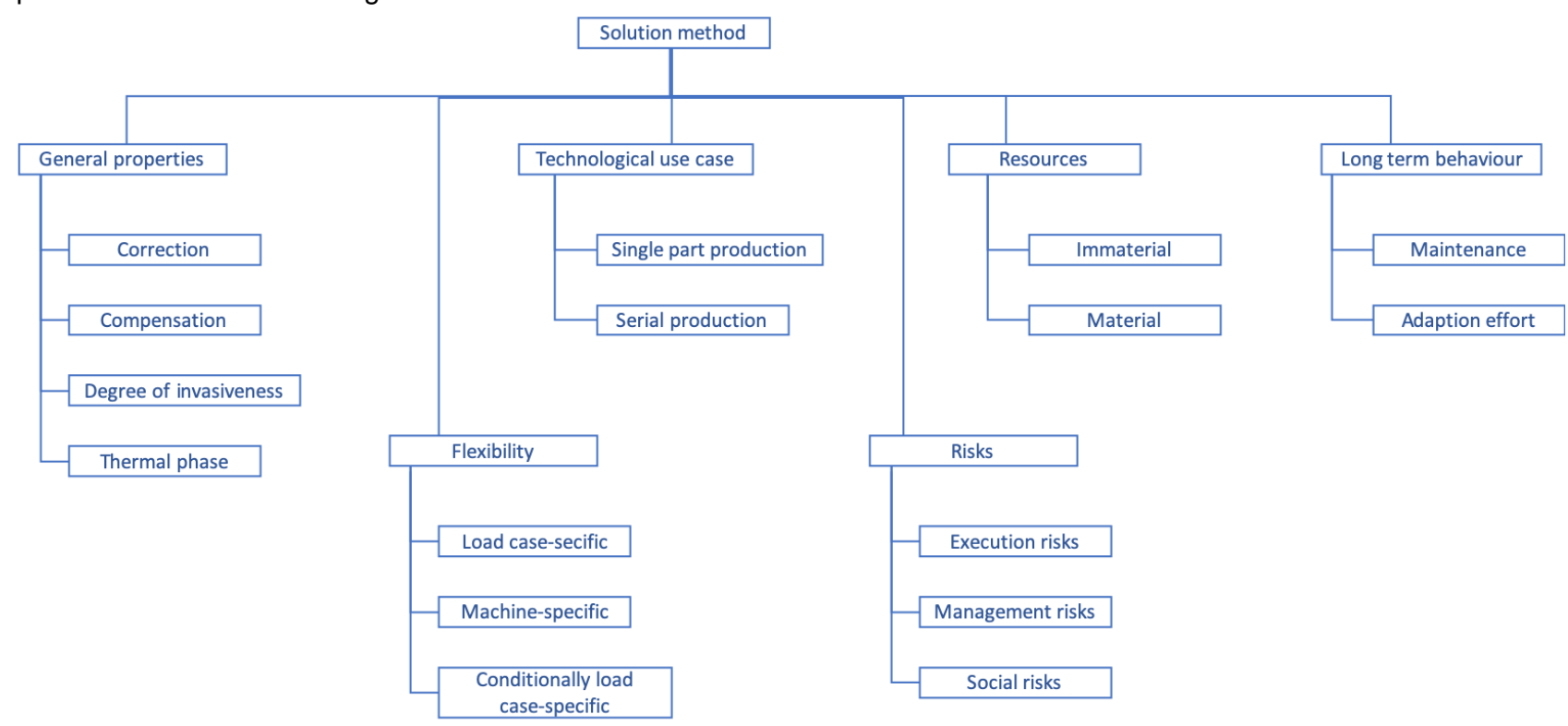

Fig. 2: Overview of the categorization of solution methods. 
For industrial applications, this means that load case specific solutions can be applied for technological use cases with repetitive thermal loads, such as series production.

The constantly varying thermal loads of a single part production require a machine-specific approach.

Furthermore, the intervention points of the methods in relation to the thermo-elastic chain of effects [Großmann 2012] can be used as a category. This makes it possible to determine which data and assumptions a method works with. For example, a method that operates in the first thermal phase " heat loss" will try to stabilise the heat input, while methods that operate in the phases "temperature field" or "deformation field" try to correct the error effect through model-based calculations by applying correction values to the machine control.

The conducted interviews also gave an overview of the resources that are necessary to implement the specific solution method. They can be divided into material and immaterial.

Immaterial resources were further divided into control licenses, special software licenses and machine downtime required for preparation and installation.

The material resources contain the material necessary for the implementation of the solution method (e.g. sensors and special components). In addition, this category includes the process steps required for the application of the solution and the time required for this. The following typical process steps were derived from the entirety of the interviews: Model design, load case definition, control integration, design, manufacturing, measurement and commissioning. Depending on the requirements of these steps for the specific solution, a distinction must be made between standard tasks that can be performed by personnel with basic training (basic hours) and those that require very specialized knowledge (hours with high know-how).

For the operating phase of the solution method, the longterm behaviour is decisive. This category describes the follow-up costs to be expected after successful commissioning. This can be caused, for example, by wear and tear on important parts or necessary modifications. The category long-term behaviour is therefore divided into maintenance and adaptation effort.

A final point to be applied in this effort assessment is the risks associated with implementing one of these solutions. The evaluation identified various keywords relating to the risk involved in the introduction or implementation of the methods, e.g. communication risks with customers, coordination risk when implementing the method on the machine, risk of wrong estimations etc. Since these risks are project development risks, which also correspond to the project character of the methods, a structure from classical risk management can be used to classify the risk of the methods. In this case, the classification of project risks according to Jenny was used, as this was defined in a generally applicable and cross-sectoral manner. A distinction is made between execution risks (implementation risk, functional risk, material supply risk), management risks (project management risk, planning risk, communication risk, coordination risk) and social risks (motivation risk, employee risk, political risk) [Jenny 2007].

The identified criteria for the categories of risks and longterm behaviour can be described using the material and immaterial resource categories mentioned above. All identified resources were evaluated individually for each solution process using a 3-point scale (low, medium, high) and cumulated. Furthermore, through process modelling it was possible to determine how much specialised know-how and expertise is needed to implement a method. This is significant because the methods are still under development and are novel in nature. From the total estimated expenditure, a portfolio was thus created which compares all nine solutions by putting the expenditure in relation to the identified expertise for the respective method (see Figure 3). As the estimated effort is based more on qualitative estimations, the portfolio is displayed twodimensionally and divided into four quadrants, which allow an assessment of the relative positioning of the different methods.

As the criteria of flexibility and technological application do not cause any direct expense and cannot be described as resource input, they were left out of this calculation.

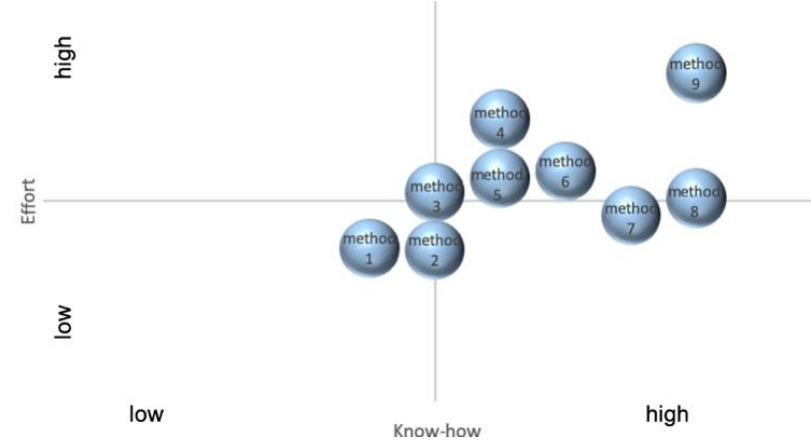

Fig. 3: Portfolio demonstrating the estimated effort in relation to the required knowledge of each method

\section{DISCUSSION}

Despite the development stage of the individual solution processes, the identified categories with the explained criteria seem to be suitable for making an initial assessment of the effort required for the individual solutions and thus, be used for a first comparison as shown in Figure 3. Moreover, although the assessment can only be made based on an abstract scale since the development process makes a quantitative assessment difficult, a comparability of the methods can be established at different levels. For example, correction methods, in contrast to compensation methods, usually require more material input, whereas compensation methods require more specialist knowledge and thus more personnel input in the model simulation. By illustrating the use of resources based on the methods modelled with BPMN, the effort involved is comprehensible and can be classified. The process steps listed in chapter three are only the steps that occur in the individual solution methods and are differently structured in the process. They could be extended by other steps of different methods that have not been considered in this work.

On the one hand, the illustration of the expenditure has made it clear that the greatest expenditure in all procedures is incurred in the installation phase of the life cycle costs, and on the other hand, the costs of the installation phase defined in the VDMA guideline are not detailed enough to provide a proper illustration of the expenditure. The effort is therefore rather the result of cumulating the individual process steps with the proposed resource input. Furthermore, this way of mapping the effort allows comparability for different application scenarios.

Additionally, as stated in chapter three the category degree of invasiveness can lead to different efforts, e.g. in terms of additional material or mandatory software licenses in order to access the machine control. This could be considered in the cumulated effort of all the method's process steps. However, this category must be considered in a more 
differentiated way since it also includes non-quantifiable aspects such as the risk of losing a machine's manufacturer's warranty or becoming dependent on a manufacturer's software.

For the categories risks and long-term behaviour, no meaningful assessments are currently possible. Rather, an assessment based on the expected expenditure must be used here. However, the criteria of the risk category already allow for comparability at the level of method type. It is already foreseeable, for example, that compensation methods that rely on external components that must be installed in the machine will have more risks in management and implementation, whereas correction methods are more likely to have risks in design and installation.

No detailed benefit can yet be compared with the expenditure shown. It is true that initial statements have already been made about the flexibility and suitability for specific applications. However, these are only estimates based on the interview data.

Yet, the categories identified have made it possible to carry out an internal analysis of the individual procedures and to provide an outlook on strengths and weaknesses as well as the core competencies of the methods. Future methods can also be classified in this classification. As the assessment scale becomes more quantitative with an advanced project status, but the criteria remain the same, it is possible to classify methods independently of their current development status.

\section{OUTLOOK}

Since, as already mentioned, the benefit of the methods could not yet be assessed, a concrete metrological implementation of the application suitability should make the benefits of the individual procedures more quantifiable. Additionally, the long-term behaviour of the solution methods shall be further investigated and defined in more detail. Therefore, the specification of the VDMA guideline 34160 can serve as an orientation.

In addition, the know-how identified should be set in relation to the Technology Readiness Level of the respective method. As the development of the method progresses, the current assessment may change, for example, if less expertise is required and the effort required is therefore put into a different perspective.

If this additional information is available, it is possible to derive success factors for the individual methods and thus provide a basis for transferring the knowledge to industrial usage.

For a potential user who wants to use one of the examined methods, it is important to know what the advantages and disadvantages of the method are compared to the other alternatives. It is not possible to make a blanket statement based purely on the amount of effort involved, nor is it solely possible to determine the suitability of the method by measuring it. Instead, a user-specific assessment must be made which considers the user's preferences and initial operational situation. For this purpose, the collected effort must be differentiated into individual categories to enable the user to compare the criteria with each other. Although the non-quantifiable criteria such as flexibility and technological application could not be directly estimated in terms of direct costs, they must be considered in the user's decision-making process as they could represent subjective preferences and thus can significantly influence a decision in favour of one method over another. This concerns also the degree of invasiveness. For this reason, a software-based application is to be created which offers a user the possibility of defining an individual starting position with the possibility to set inclusion and exclusion criteria and weighting the criteria according to personal preferences. Based on this, suitable solution methods should be suggested which meet the user's requirements.

In this work, the starting point was the view of a user of machine tools and correspondingly adjustments to existing processes. A manufacturer, who has different circumstances than a user and can start at a different process step to improve the productivity of machine tools, needs different resources and input. Taking this perspective into account would therefore be another interesting starting point for the comparative evaluation of solution methods.

\section{CONCLUSION}

In research, a variety of solution methods exist to correct or compensate thermally induced displacements.

These differ strongly in their approaches, requirements, and their implementation on machine tools.

In combination with the large variety of machine structures and applications, it is often difficult for potential users to select a suitable solution and to estimate the effort for the implementation on existing machine tools.

A direct comparison of the solution methods is rarely possible.

In this paper, a taxonomy was developed based on expert interviews on nine different approaches to correct thermally induced errors.

By comparing the necessary steps and resources to implement the solutions it was possible to derive categories.

Portfolios for all solution methods were created from the total implementation effort.

Based on these portfolios it was finally possible to compare the different solutions directly with each other.

This work represents an important step to ensure the transfer of the solution methods from research to industry. In further work, it will be necessary to quantify the effect of the solutions on machine tools.

Once this data is available, the derivation of success factors and thus ultimately a user-specific recommendation is possible.

\section{ACKNOWLEDGMENTS}

This research was funded by the German Research Foundation - Project-ID 174223256- TRR 96, which is gratefully acknowledged.

\section{REFERENCES}

[Braun 2014] Braun, R. and Esswein, W. Extending BPMN for modelling resource aspects in the domain of machine tools. In: Proceedings of the International Conference on Advanced Materials and Information Technology Processing, Los Angeles, 1 February, 2014. WIT Press, pp 449458, ISSN 17464471

[Bryan 1968] Bryan, J. International Status of Thermal Error Research. In: CIRP Annals, Volume 16, 1968, pp203215

[Bryan 1990] Bryan, J. International Status of Thermal Error Research. In: CIRP Annals, Volume 39, Issue 2, 1990, pp645656, ISSN 0007-8506 
[Großmann 2012] Großmann, K. Thermo-Energetische Gestaltung von Werkzeugmaschinen, Eine Übersicht zu Zielen und Vorgehen im SFB/Transregio 96. In: Zeitschr. f. wirtsch. Fabrikbetrieb, Volume 05, 2012, pp. 307314

[Großmann 2015] Großmann, K. et al. Thermo-energetic Design of Machine Tools, A systemic approach to solve the conflict between power efficiency, accuracy and productivity demonstrated at the example of machining production. Springer International Publishing, Switzerland, 2015, ISSN 2194-0525

[Jenny 2007] Jenny, B. Project Management: Knowledge for a Successful Career. Zurich: vdf, 2007.

[Kirchner 2014] Kirchner, K. et al. Experience from collaborative modelling of clinical pathways. In: Hess, M. and Schlieter, H., eds. ICB Research Report, March, 2014, Essen: ICB, pp. 1324, ISSN 18602770

[Mayr 2012] Mayr, J. et al. Thermal issues in machine tools. In: CIRP Annals, Volume 61, Issue 2, 2012, pp. 771779, ISSN 0007-8506
[Mayr 2017] Mayr, J. Thermal error research, an overview. In: Laser Metrology and Machine Performance XII, Volume 12, 2017, United Kingdom, pp. 1031

[Mayring 2014] Mayring, P. Qualitative content analysis: theoretical foundation, basic procedures and software solution. Klagenfurt, 2014.

[Putz 2018] Putz, M. et al. Industrial consideration of thermal issues in machine tools. In: Production Engineering, Volume 12, Issue 6, 2018, pp. 723736

[VDMA 2006] Verband Deutscher Maschinen- und Anlagenbau e.V. VDMA 34160 Prognosemodell für die Lebenszykluskosten von Maschinen und Anlagen. Beuth Verlag, Berlin, June, 2006

[Weck 1995] Weck, M. et al. Reduction and Compensation of Thermal Errors in Machine Tools. In: CIRP Annals, Volume 44, Issue 2, 1995, pp. 589598, ISSN 0007-8506,

[Wegener 2016] Wegener, K. et al. Age of Compensation Challenge and Chance for Machine Tool Industry. In: Int. J. Automation Technol., Vol.10, No.4, pp. 609-623, 2016, : 